\title{
SARS-CoV-2 vaccine-related neurological complications
}

\author{
Nicol G. M. Oonk ${ }^{1} \cdot$ Anne Rozemarijn Ettema ${ }^{1} \cdot$ Heleen van Berghem ${ }^{1} \cdot$ Jabke J. de Klerk $^{2} \cdot$ Joyce P. M. van der Vegt $^{1}$. \\ Matthijs van der Meulen ${ }^{1}$
}

Received: 24 May 2021 / Accepted: 13 January 2022 / Published online: 20 January 2022

(c) Fondazione Società Italiana di Neurologia 2022

\begin{abstract}
Objective To describe three cases with neurological symptoms after SARS-CoV-2 vaccination.

Methods A case series followed by a review of the literature, describing hypotheses on how neurological symptoms might develop after vaccination.

Results The different temporal relationship between the onset or worsening of different neurological symptoms suggests different pathophysiological mechanisms. Progression of post-infectious myoclonus, caused by a previous SARS-CoV2-infection, shortly after vaccination suggests a renewed auto-immune mediated crossreaction of antibodies to both viral epitopes and central nervous system components. Thunderclap headache after vaccination suggests a similar pathophysiological mechanism to the headache and other flu-like symptoms described after vaccination against other viruses. This might be ascribed to the activation of immunoinflammatory mediators or accompanying fever. Although headache accompanied by encephalopathy and focal neurological deficit might occur as part of a cytokine release syndrome, this is clinically less likely. Conclusions A variety of symptoms, including thunderclap headache, focal deficits and movement disorders, can occur after SARS-CoV-2 vaccination, and an activation or reactivation of the immune system is suggested as most likely cause. However, one should be careful about claiming a direct correlation. It remains important to exclude other causes, such as structural lesions, infections or subarachnoid hemorrhage, and future research is required to understand possible pathophysiological mechanisms and associations with the SARS-CoV-2 vaccine.
\end{abstract}

Keywords SARS-CoV-2 $\cdot$ COVID-19 $\cdot$ Vaccine $\cdot$ Thunderclap headache $\cdot$ Myoclonus

\section{Introduction}

Worldwide, many people have been infected with SARS$\mathrm{CoV}-2$, which has resulted in morbidity and mortality [1]. This pandemic is still having an enormous impact on healthcare and society in general [2]. Vaccination against SARS-CoV-2 is important to reduce spread of the virus and death rates. Although multiple neurological signs and symptoms in patients with a SARS-CoV-2 infection have been reported [1], the information about neurological complications after SARS-CoV-2 vaccination is also increasing [3]. With this case series, we aim to contribute to this knowledge by describing three cases with neurological manifestations

Nicol G. M. Oonk

n.oonk@mst.nl

1 Department of Neurology, Medisch Spectrum Twente, Enschede, the Netherlands

2 TriviumMeulenbeltZorg, Borne, the Netherlands after vaccination against SARS-CoV-2. All patients gave written informed consent. Adverse effects were registered in the national pharmacovigilance center (Lareb).

\section{Case A}

An 87-year-old man known with hypertension had a SARSCoV-2 infection in November 2020, diagnosed with a PCR-test. Thirteen days after the initial and mild flu-like symptoms, he developed a bilateral disabling, progressive, generalized, actioninduced myoclonus with a mild dysarthria, without any other symptoms. Laboratory tests showed no metabolic cause, brain MRI did not show any structural lesions, and CSF analysis showed no abnormalities, including a negative SARS-CoV-2 PCR. Also, anti-neuronal antibodies in serum were negative.

Because of the subacute onset and unremarkable ancillary investigations, a SARS-CoV-2 associated para-infectious myoclonus was diagnosed. Treatment with levetiracetam, 
clonazepam, and pulse therapy methylprednisolone was initiated. Three months after initial presentation, only a mild actioninduced myoclonus persisted. Two months after primary infection, he was vaccinated against SARS-CoV-2 with the Pfizer/ BioNTech vaccine [4]. One day after the first vaccine, there was a progression of myoclonic symptoms, with improvement within days. No worsening of symptoms occurred after the second vaccine (30 days after his first vaccine).

\section{Case B}

A 62-year-old woman with a medical history of ocular melanoma experienced a thunderclap headache without any other complaints, suddenly after her SARS-CoV-2 vaccine (Pfizer/ BioNTech) [4]. The headache spontaneously improved after one day. Ten days after vaccination, she had a sudden brief loss of consciousness without head trauma, directly followed by a second episode of thunderclap headache. Neurological examination showed a bradyphrenic woman with motoric dysphasia and mild dysmetria in all extremities, and was otherwise normal. Laboratory analysis, brain CT and MRI, EEG and CSF analysis including blood pigment and cytology analysis were all unremarkable. Cardiological work-up showed no abnormalities. No cause could be found for the headache, including no signs of cerebral reversible vasoconstriction syndrome. Her symptoms recovered within a few days.

After the second vaccination, she experienced another episode of thunderclap headache, without any other neurological deficits.

\section{Case C}

A 21-year-old woman with an unremarkable medical history developed general malaise with subfebrile temperature two hours after her first vaccination against SARS-CoV-2 (Oxford/AstraZeneca) [5]. Six hours later, she experienced a thunderclap headache, with nausea and vomiting. She had tachycardia and hypertension and was restless. Neurological examination, blood analysis, and brain CT including CTangiography and venography were all normal. The patient was treated with paracetamol, NSAIDs, intravenous morphine, and oxygen therapy. The headache diminished within $24 \mathrm{~h}$. She recovered completely within a few days.

\section{Discussion}

The pathophysiological mechanisms for the occurrence of neurological symptoms after SARS-CoV-2 vaccination are not entirely clear. The origin of a para-infectious phenomenon, as suspected in case A, is thought to be autoimmune mediated and presumably based on molecular mimicry between epitopes of viral spike proteins and specific homologous self-proteins of the nervous system [6]. Antibodies produced by the activated immune system will bind to viral epitopes and additionally, via a cross-reaction, damage specific parts of the nervous system. The modified RNA within the Pfizer/BioNTech vaccine will be anchored in the host cell membrane [4]. After vaccination, crossreactivity may occur again by renewed production of the auto-antibodies already known by the immune system [7]. We hypothesize that the relative short timespan between the first and second vaccination with a still activated immune response might explain why symptoms did not worsen after the second vaccine in case A. A recent small $(n=15)$ postmortem cohort-study showed, however, that patients with a SARS-CoV-2 infection-vaccination status unknown-have significantly more microglial activation (innate immune system) in the brainstem. This suggests that also other pathophysiological mechanisms might be involved [8].

The post-vaccination thunderclap headache, without a preceding SARS-CoV-2 infection, (case B and C) presumably, has a different cause. The onset of symptoms shortly after vaccination suggests a direct correlation with SARS-CoV-2 vaccination. Different hypotheses have been postulated.

First, headache is commonly seen in patients suffering from a respiratory virus and after a vaccination, including SARS-CoV-2-vaccines [3, 4]. Post-vaccination headache might be based on the same pathophysiological mechanism as described after a virus infection: although not fully understood, this might be ascribed to fever, activation of immunoinflammatory mediators, or a direct effect of specific microorganisms [9]. This might also apply to post-vaccination thunderclap headache, supported by the concomitant signs of malaise and subfebrile temperature (case C).

Second, there might be reactivation of the immune system when patients have previously been infected with SARSCoV-2 or a SARS-related infection. Medical history of patient $\mathrm{B}$ and $\mathrm{C}$ did not reveal this, but symptoms may have been subclinical.

Third, headache and encephalopathy have been reported as a neurological manifestation of SARS-CoV-2-infection due to virus induced systemic hyperinflammation by an extensive cytokine release [10]. This could cause inflammation of multiple organ systems, including the central nervous system, and might lead to multi-organ failure [11]. However, since the symptoms were relatively mild and the patients in case $B$ and $C$ were only vaccinated without a preceding infection, this cause is unlikely. Hypothetically, a milder version of this phenomenon could have occurred.

To date, there is no evidence for higher rates of neurological disease after COVID-19 vaccination [12] and it remains 
therefore unclear whether the episode of bradyphrenia and focal neurological symptoms could be related to the SARS$\mathrm{CoV}-2$ vaccine.

It remains essential to exclude other underlying conditions, including subarachnoid hemorrhage, stroke, cervical artery dissection, and cerebral venous sinus thrombosis.

\section{Conclusion}

This case series show new onset or deterioration of neurological symptoms shortly after the administration of a SARS-CoV-2 vaccine. Although a causal relationship could not be established, the temporal relationship between vaccination and symptoms, the exclusion of other causes of the signs and symptoms after thorough examination, and the known pathophysiological mechanisms as described support our hypotheses. In sum, after vaccination, a (re)activation of the immune system might cause neurological symptoms, but it remains important to exclude other etiologies. Further research may gain more insights into the mentioned hypotheses.

Author contribution Acquisition of data: NGM Oonk, AR Ettema, H van Berghem, JJ de Klerk, JPM van der Vegt, M van der Meulen; design and conceptualized study: NGM Oonk, AR Ettema; M van der Meulen; interpreted the data: NGM Oonk, AR Ettema, JPM van der Vegt, $M$ van der Meulen; drafted the manuscript for intellectual content: NGM Oonk, AR Ettema; M van der Meulen; revised the manuscript for intellectual content: $\mathrm{H}$ van Berghem, JJ de Klerk, JPM van der Vegt.

\section{Declarations}

Ethical approval The study was approved by the institutional review board.

Consent to participate Informed consent was obtained from all participants included in the study.

Conflict of interest The authors declare no competing interests.

\section{References}

1. Romero-Sánchez CM, Díaz-Maroto I, Fernández-Díaz E et al (2020) Neurologic manifestations in hospitalized patients with COVID-19: The ALBACOVID registry. Neurology 95:e1060e1070. https://doi.org/10.1212/WNL.0000000000009937

2. van der Meulen M, Kleineberg NN, Schreier DR et al (2020) COVID-19 and neurological training in Europe: from early challenges to future perspectives. Neurol Sci 41:3377-3379. https:// doi.org/10.1007/s10072-020-04723-9

3. Patone M, Handunnetthi L, Saatci D et al (2021) Neurological complications after first dose of COVID-19 vaccines and SARSCoV-2 infection. Nat Med 27:2144-2153. https://doi.org/10.1038/ s41591-021-01556-7

4. Polack FP, Thomas SJ, Kitchin N et al (2020) Safety and efficacy of the BNT162b2 mRNA Covid-19 vaccine. N Engl J Med 383:2603-2615. https://doi.org/10.1056/nejmoa2034577

5. Voysey M, Clemens SAC, Madhi SA et al (2021) Safety and efficacy of the ChAdOx1 nCoV-19 vaccine (AZD1222) against SARS-CoV-2: an interim analysis of four randomised controlled trials in Brazil, South Africa, and the UK. Lancet 397:99-111. https://doi.org/10.1016/S0140-6736(20)32661-1

6. Albert LJ, Inman RD (1999) Molecular mimicry and autoimmunity. N Engl J Med 341:2068-2074. https://doi.org/10.1056/nejm1 99912303412707

7. Segal Y, Shoenfeld Y (2018) Vaccine-induced autoimmunity: the role of molecular mimicry and immune crossreaction. Cell Mol Immunol 15:586-594. https://doi.org/10.1038/cmi.2017.151

8. Poloni TE, Medici V, Moretti M et al (2021) COVID-19-related neuropathology and microglial activation in elderly with and without dementia. Brain Pathol 31:1-16. https://doi.org/10.1111/bpa. 12997

9. Headache Classification Committee of the International Headache Society (2018) The International Classification of Headache Disorders, 3rd edition. Cephalalgia 38:1-211. https://doi.org/10.1177/ 0333102417738202

10. Perrin P, Collongues N, Baloglu S et al (2021) Cytokine release syndrome-associated encephalopathy in patients with COVID-19. Eur J Neurol 28:248-258. https://doi.org/10.1111/ene.14491

11. Koralnik IJ, Tyler KL (2020) COVID-19: a global threat to the nervous system. Ann Neurol 88:1-11. https://doi.org/10.1002/ana. 25807

12. Goss AL, Samudralwar RD, Das RR, Nath A (2021) ANA investigates: neurological complications of COVID-19 vaccines. Ann Neurol 89:856-858. https://doi.org/10.1002/ana.26065

Publisher's Note Springer Nature remains neutral with regard to jurisdictional claims in published maps and institutional affiliations. 